\title{
Experiência de estudantes de enfermagem na técnica de punção venosa periférica com e sem o uso de transiluminador cutâneo portátil
}

\author{
Experience of nursing students in peripheral venous puncture technique with and without the use \\ of portable skin transillumillator
}

Experiencia de estudiantes de enfermería en la técnica de punción venosa periférica con y sin el uso de transiluminador de piel portátil

Recebido: 08/08/2021 | Revisado: 14/08/2021 | Aceito: 18/08/2021 | Publicado: 22/08/2021

\author{
Hellen Cristine de Lima \\ ORCID: https://orcid.org/0000-0002-7724-2345 \\ Centro Universitário Santa Cruz de Curitiba, Brasil \\ E-mail: hellen.lima2315@gmail.com \\ Bruna Eloise Lenhani \\ ORCID: https://orcid.org/0000-0002-6009-3400 \\ Centro Universitário Santa Cruz de Curitiba, Brasil \\ E-mail: bruna.lenhani@unisantacruz.edu.br \\ Josemar Batista \\ ORCID: https://orcid.org/0000-0001-9838-1232 \\ Centro Universitário Santa Cruz de Curitiba, Brasil \\ E-mail: josemar.batista@unisantacruz.edu.br \\ Catia Terezinha Heimbecher \\ ORCID: https://orcid.org/0000-0003-4487-153X \\ Centro Universitário Santa Cruz de Curitiba, Brasil \\ E-mail: catia.heimbecher@unisantacruz.edu.br
}

\section{Resumo}

Objetivo: Descrever a experiência de acadêmicos de enfermagem na técnica de punção venosa periférica com e sem o uso de transiluminador cutâneo portátil. Método: Estudo observacional, descritivo, transversal e quantitativo realizado em uma faculdade privada no município de Curitiba-Paraná. Participaram 18 acadêmicos divididos aleatoriamente em dois grupos: Grupo I (técnica tradicional, sem o uso transiluminador cutâneo portátil - venoscópio) e Grupo II (técnica com o uso do venoscópio). Os dados foram coletados com a utilização de dois instrumentos elaborados pelos pesquisadores contendo variáveis demográficas e questões relacionadas ao uso do venoscópio. Utilizou-se de estatística descritiva e inferencial para a análise dos dados. Resultados: Houve correlação significativa entre o conhecimento teórico de punção venosa e a identificação do vaso a ser puncionado ( $\mathrm{p}=0,000)$, e do conhecimento teórico com o nível de dificuldade para puncionar $(\mathrm{p}=0,005)$. Quanto ao número de tentativas de punção, 33,3\% dos acadêmicos puncionaram com êxito na primeira tentativa com o venoscópio, para 44,4\% dos acadêmicos sem o uso do aparelho. Ao abordar a experiência com o uso do venoscópio, 66,7\% citaram ser vantajosa, e 55,6\% relataram que o seu uso favoreceu a visualização de veias. Conclusão: A punção venosa com o uso do venoscópio foi válida ao aprendizado e considerada uma experiência vantajosa para os acadêmicos.

Palavras-chave: Estudantes de enfermagem; Transiluminação; Cateterismo periférico; Ensino; Educação em enfermagem.

\begin{abstract}
Objective: To describe the experience of nursing students in the technique of peripheral venous puncture with and without the use of portable cutaneous transillator. Method: Observational, descriptive, cross-sectional and quantitative study conducted in a private college in the city of Curitiba-Paraná. Participants were 18 students randomly divided into two groups: Group I (traditional technique, without the use of portable skin transilluminator - venoscope) and Group II (technique with the use of venoscope). Data were collected using two instruments developed by the researchers containing demographic variables and questions related to venoscope use. Descriptive and inferential statistics were used for data analysis. Results: There was a significant correlation between the theoretical knowledge of venous puncture and the identification of the vessel to be punctured $(\mathrm{p}=0.000)$, and the theoretical knowledge with the level of difficulty to puncture $(\mathrm{p}=0.005)$. Regarding the number of puncture attempts,33.3\% of the students successfully punctured the first attempt with the venoscope, for $44.4 \%$ of the students without the use of the device. When addressing the experience with the use of the venoscope, $66.7 \%$ mentioned being advantageous, and $55.6 \%$ reported that its use favored the visualization of veins. Conclusion: Venous puncture with the use of the venoscope was valid for learning and considered an advantageous experience for the students.
\end{abstract}

Keywords: Students nursing; Transillumination; Catheterization peripheral; Teaching; Education nursing. 


\begin{abstract}
Resumen
Objetivo: Describir la experiencia de los estudiantes de enfermería en la técnica de punción venosa periférica con y sin el uso de transiluminador cutáneo portátil. Método: Estudio observacional, descriptivo, transversal y cuantitativo realizado en un colegio privado de la ciudad de Curitiba-Paraná. Los participantes fueron 18 estudiantes divididos aleatoriamente en dos grupos: Grupo I (técnica tradicional, sin el uso de transiluminador de piel portátil - venoscópio) y Grupo II (técnica con el uso de venoscópio). Los datos fueron recolectados utilizando dos instrumentos desarrollados por los investigadores que contenían variables demográficas y preguntas relacionadas con el uso del venoscópio. Para el análisis de los datos se utilizó estadística descriptiva e inferencial. Resultados: Hubo correlación significativa entre el conocimiento teórico de la punción venosa y la identificación del vaso a perforar $(\mathrm{p}=0,000)$, y el conocimiento teórico con el nivel de dificultad para la punción $(\mathrm{p}=0,005)$. En cuanto al número de intentos de punción, el 33,3\% de los estudiantes perforó con éxito el primer intento con el venoscópio, para el 44,4\% de los estudiantes sin el uso del dispositivo. Al abordar la experiencia con el uso del venoscópio, 66,7\% mencionaron ser ventajoso, y 55,6\% relataron que su uso favoreció la visualización de venas. Conclusión: La punción venosa con el uso del venoscópio fue válida para el aprendizaje y considerada una experiencia ventajosa para los estudiantes.

Palabras clave: Estudiantes de enfermería; Transiluminación; Cateterismo periférico; Enseñanza; Educación en enfermería.
\end{abstract}

\title{
1. Introdução
}

A punção venosa periférica (PVP) é caracterizada como um procedimento crítico e invasivo em que ocorre a instalação de um dispositivo estéril no interior do vaso (Alves et al., 2019). É considerada uma atividade rotineira e complexa no exercício da profissão de enfermagem, o que, por sua vez, demanda competências específicas em todo o processo (Caramelo et al., 2019), principalmente para antever os riscos e complicações oriundos do procedimento.

Ressalta-se que o insucesso na PVP contribui para a ocorrência de complicações tais como flebite, infiltração, hematoma, trombose e tromboflebite (Mota et al., 2019), agravadas em detrimento da presença de pacientes com rede venosa de difícil cateterização. Uma revisão integrativa apontou a prevalência da PVP difícil entre 17\% e 59,3\% estando associadas a fatores demográficos (sexo e idade), clínicos (comorbidades, estado nutricional, visibilidade e palpabilidade da rede venosa), do dispositivo (calibre e modelo) e à habilidade do profissional (Marinho et al., 2019).

Desta forma, frente aos riscos e complicações e dos fatores que contribuem para o sucesso da PVP, cabe aos profissionais de saúde, em especial, da enfermagem, seguir diretrizes clínicas recomendadas por órgãos nacionais e internacionais. O Centers for Disease Control and Prevention (O'grady et al., 2011), o Royal College of Nursing (Dougherty et al., 2010) e a Infusion Nurses Society (Gorski et al., 2016), são exemplos de instituições que orientam cuidados específicos no que tange a utilização de tecnologias que auxiliem na visualização da veia, tal qual o uso do venoscópio (transiluminador cutâneo portátil) durante a inserção de cateteres venosos periféricos (CVP). Para garantir a segurança do paciente, a utilização dessa tecnologia é indicada para pacientes adultos e pediátricos com acesso venoso difícil e/ou após várias tentativas de PVP malsucedidas (Gorski et al., 2016).

No entanto, a literatura revela que inserir o CVP com sucesso na primeira tentativa reduz o desconforto para o paciente e consequentemente, contribui para diminuir a frustração do profissional de saúde e os custos associados a esse procedimento (Marinho et al., 2019). Por outro lado, verificou-se em estudo conduzido com 26 enfermeiros atuantes em hospital de Portugal que as práticas relacionadas com CVP não respeitaram as atuais recomendações/guidelines nacionais e internacionais de padrões de cuidados, incluindo a não utilização de tecnologia de visualização vascular (por exemplo, ultrassom ou luz quase-infravermelha) para auxiliar em casos complicados, uma vez que estas tecnologias não existiam nas unidades investigadas (Oliveira et al., 2019).

Por se tratar de uma tecnologia inovadora, esses dados mostram a importância do apoio da gestão para o uso cotidiano desta tecnologia no cuidado de enfermagem e a necessidade de fomentar as instituições de ensino a utilizá-la durante a formação inicial do profissional enfermeiro. No futuro, a assimilação dessa tecnologia irá contribuir para aumentar a satisfação desses profissionais, impactando diretamente na qualidade e segurança na saúde (Oliveira et al., 2016). 
A eficácia no desempenho para a realização da técnica de PVP requer conhecimentos e habilidades adquiridas no decorrer do curso mediante as atividades teóricas e práticas, sendo estes requisitos básicos para os profissionais de saúde que realizam procedimentos complexos (Mota et al., 2019).

Frente a necessidade de introduzir novas tecnologias no processo de ensino e aprendizagem dos profissionais de enfermagem para ofertar assistência com mais segurança e menos riscos, justifica-se a presente pesquisa que teve como questão norteadora: como foi a experiência de acadêmicos de enfermagem na técnica de punção venosa periférica com e sem o uso de transiluminador cutâneo portátil?

O objetivo da presente pesquisa foi descrever a experiência de acadêmicos de enfermagem na técnica de punção venosa periférica com e sem o uso de transiluminador cutâneo portátil.

\section{Metodologia}

Estudo observacional, descritivo e transversal de abordagem quantitativa. Foi realizado no Laboratório de habilidades em Semiologia e Semiotécnica de uma Faculdade privada no município de Curitiba-Paraná, no período de agosto a setembro de 2019. A população do estudo foi composta por 23 acadêmicos matriculados no quarto período do curso de graduação em Enfermagem.

Foi considerado como critério de inclusão não possuir qualquer conhecimento prático relacionado a técnica de PVP. Excluíram-se os estudantes menores de 18 anos de idade e os técnicos de enfermagem. Após a aplicação dos critérios e do convite individual com esclarecimentos pertinentes ao estudo, 18 acadêmicos aceitaram participar da pesquisa.

Para a condução da prática simulada em laboratório de habilidades, os acadêmicos participaram de uma aula expositiva e dialogada, com duração de 60 minutos, na qual foram abordados princípios da PVP. Na sequência, os acadêmicos foram divididos aleatoriamente em dois grupos: Grupo I (técnica tradicional, sem o uso do transiluminador cutâneo portátil venoscópio) e Grupo II (técnica com o uso do venoscópio). Cabe destacar, que para organizar a divisão dos acadêmicos foi utilizada a lista de frequência da turma em ordem alfabética e numerada em ordem crescente. Os acadêmicos com números ímpares foram alocados no Grupo II, referente a PVP com utilização do uso do transiluminador cutâneo portátil.

Para o método de PVP tradicional (Grupo I), foi utilizado o garroteamento com luva de procedimento de $10 \mathrm{a} 15 \mathrm{~cm}$ acima da incisão do local a ser puncionado, e o papel toalha alocado abaixo do garrote ou uma camada fina de roupa a fim de não lesionar a pele do participante. A veia foi localizada com a ajuda do tato, realizada a antissepsia da pele com algodão embebido em álcool a 70\%, com inserção da agulha do cateter flexível número 22, com o bisel para cima em ângulo de 10 a 30 graus. Após esse procedimento, constatou-se o retorno venoso na câmara do dispositivo. O cateter foi abaixado até nivelá-lo com a pele sendo introduzido até que o conector estivesse próximo ao local de inserção. Por fim, soltou-se o garrote, verificando a ausência de formação de inchaço com a infusão de cloreto de sódio e realizada a fixação do cateter com fita micropore, deixando descoberta a conexão entre o equipo e o conector (Potter, 2017).

O método de PVP com o auxílio do transiluminador cutâneo portátil (Grupo II) foi realizado de maneira semelhante ao tradicional. Contudo, a procura do vaso não foi realizada por meio do tato, mas com o direcionamento da luz infravermelha sobre a pele. Nesta pesquisa o aparelho foi posicionado diretamente sobre a pele do participante. Foi utilizado o aparelho de transiluminador cutâneo portátil VF 1000 LED $^{\circledR}$ (Light-emitting diode), localizador de veias. O aparelho é manual, portátil e não invasivo. Utiliza lâmpadas de LED como mecanismo refletor aos componentes sanguíneos, proporcionando uma visualização privilegiada dos vasos pelo acadêmico.

Para a coleta de dados, foram elaborados dois instrumentos pelos pesquisadores. O primeiro questionário foi preenchido pelo próprio acadêmico e continha 10 questões, duas referentes a idade e sexo; as demais relacionadas com a experiência vivenciada ao realizar a PVP. O segundo instrumento foi preenchido pela própria pesquisadora e continha questões 
relacionadas a avaliação da punção realizada pelo acadêmico, como: veia escolhida e número de tentativas para atingir a técnica correta.

Os dados coletados foram tabulados com auxílio do programa Microsoft Excel ${ }^{\circledR} 2007$, e processados pelo Statistical Package for the Social Science ${ }^{\circledR}$ (SPSS), versão 21.0. Utilizou-se a estatística descritiva para analisar os dados. Aplicou-se a análise de variância (ANOVA) para as variáveis categóricas. O nível de significância adotado foi de p $\leq 0,05$. Esta pesquisa atendeu aos preceitos éticos da Resolução 466/2012 e foi aprovada pelo Comitê de Ética em Pesquisa institucional sob parecer número 3.483.401 de 02 de agosto de 2019.

\section{Resultados}

Participaram 18 acadêmicos de enfermagem e a média de idade encontrada foi de 25 anos (desvio padrão - DP \pm 8,47), com variação entre 18 e 47 anos. Houve prevalência de acadêmicos do sexo feminino (94,4\%; n=17), e correlação significativa entre o conhecimento teórico de punção venosa e a identificação do vaso a ser puncionado ( $\mathrm{p}=0,000)$, assim como sobre este conhecimento e o nível de dificuldade para puncionar $(\mathrm{p}=0,005)$.

Os conhecimentos dos acadêmicos acerca da técnica de PVP e ao que se refere ao uso ou não do transiluminador cutâneo portátil foram apresentados utilizando variáveis como conhecimento da técnica de punção e do transiluminador cutâneo, facilidade ou não na identificação da veia, prática válida ao aprendizado e se a experiência foi vantajosa, assim como, se houve dificuldades no processo (Tabela 1).

Tabela 1. Distribuição das variáveis de aprendizagem com ou sem o uso do transiluminador cutâneo portátil por acadêmicos de enfermagem. Curitiba, PR, Brasil, 2019.

\begin{tabular}{|c|c|c|c|c|c|c|c|c|}
\hline \multirow{3}{*}{ Variáveis } & \multicolumn{4}{|c|}{$\begin{array}{c}\text { Com transiluminador cutâneo } \\
\text { portátil }(n=9)\end{array}$} & \multicolumn{4}{|c|}{$\begin{array}{c}\text { Sem transiluminador cutâneo } \\
\text { portátil }(n=9)\end{array}$} \\
\hline & \multicolumn{2}{|c|}{ Sim } & \multicolumn{2}{|c|}{ Não } & \multicolumn{2}{|c|}{ Sim } & \multicolumn{2}{|c|}{ Não } \\
\hline & $\mathrm{n}$ & $\%$ & $\mathrm{n}$ & $\%$ & $\mathrm{n}$ & $\%$ & $\mathrm{n}$ & $\%$ \\
\hline Conhece a técnica de punção & 6 & 66,7 & 3 & 33,3 & 5 & 55,6 & 4 & 44,4 \\
\hline Conhece o transiluminador cutâneo portátil e função & 7 & 77,8 & 2 & 22,2 & 6 & 66,7 & 3 & 33,3 \\
\hline Identificou a veia com facilidade & 5 & 55,6 & 4 & 44,4 & 4 & 44,4 & 5 & 55,6 \\
\hline Prática válida ao aprendizado & 7 & 77,8 & 2 & 22,2 & 7 & 77,8 & 2 & 22,2 \\
\hline Experiência vantajosa & 6 & 66,7 & 3 & 33,3 & - & - & - & - \\
\hline Dificuldade no procedimento & 5 & 55,6 & 4 & 44,4 & 6 & 66,7 & 3 & 33,3 \\
\hline
\end{tabular}

Fonte: Autores.

Quanto ao número de tentativas de punção até o acerto da técnica, 33,3\% (n=3) realizaram a punção venosa periférica na primeira tentativa com o transiluminador cutâneo portátil, enquanto $44,4 \%(n=4)$ dos acadêmicos o fizeram sem o uso do aparelho. Os demais acadêmicos realizaram a punção na segunda tentativa em ambos os grupos. Os vasos de escolha pelos acadêmicos de enfermagem para realização da PVP foram a veia basílica, mediana e cefálica (Tabela 2). 
Tabela 2. Distribuição da escolha do vaso a ser puncionado pelos acadêmicos de enfermagem com ou sem o uso do transiluminador cutâneo portátil. Curitiba, PR, Brasil, 2019.

\begin{tabular}{lcccc}
\hline \multirow{2}{*}{ Veia } & \multicolumn{2}{c}{ Com transiluminador cutâneo portátil } & \multicolumn{2}{c}{$\begin{array}{c}\text { Sem transiluminador cutâneo portátil } \\
(\mathbf{n = 9})\end{array}$} \\
\cline { 2 - 5 } & $\mathrm{n}$ & $\%$ & $\mathrm{n}$ & $\%$ \\
\hline Basílica & 2 & 22,2 & 1 & 11,1 \\
Mediana & 5 & 55,6 & 6 & 66,7 \\
Cefálica & 2 & 22,2 & 1 & 11,1 \\
Não soube identificar & 0 & 0 & 1 & 11,1 \\
\hline
\end{tabular}

Fonte: Autores.

Para os acadêmicos que utilizaram o transiluminador cutâneo portátil para PVP, 55,6\% (n=5) acreditam que seu uso contribui para a visualização das veias e facilitou a realização do procedimento; e 44,4\% (n=4) consideraram difícil manipular ou realizar a punção venosa com o uso do aparelho.

\section{Discussão}

Os resultados apontam predominância de participantes do sexo feminino, corroborando com o perfil da enfermagem brasileira que majoritariamente é feminina (85\%) (Silva et al., 2020). O uso de tecnologias como o transiluminador cutâneo portátil utilizado nesta pesquisa, proporciona a efetividade e eficiência na PVP; entretanto, é importante primar pela habilidade do profissional em utilizá-la. Portanto, o treinamento e capacitação da equipe de enfermagem são necessários, com vistas à utilização da tecnologia e consequentemente, segurança na realização da técnica (Oliveira et al., 2016).

Com vistas a garantir qualificação profissional durante a PVP com o uso do transiluminador cutâneo portátil, faz-se necessário estimular e incluir o seu uso em escolas de enfermagem como uma das estratégias para garantir qualidade e segurança da técnica executada pelos acadêmicos de enfermagem e ao cuidado ofertado aos pacientes. A participação dos acadêmicos de enfermagem na aula expositiva e dialogada e em seguida da técnica de punção venosa com ou sem o uso da tecnologia denota a indissociabilidade entre o conhecimento teórico e a prática e o interesse no seu crescimento profissional, bem como em aprender algo novo, aplicável no futuro campo de trabalho, o qual contribui com a segurança do paciente. Associado a isto, 77,8\% dos participantes da presente pesquisa relataram ter sido válida a prática para o aprendizado, pois é uma técnica que está presente no cotidiano da prática clínica e que $70 \%$ dos pacientes hospitalizados vão precisar (Rickard et al., 2012).

Em relação ao conhecimento acerca do transiluminador cutâneo portátil, 72,2\% conheciam ou já tinham escutado falar sobre esta tecnologia mesmo sem ter feito uso da mesma. Esse achado é inferior ao estudo realizado por Soares (2018) com 110 enfermeiros especialistas das áreas de estudos da pele em que 85,5\% (n=94) dos profissionais relataram o conhecimento da tecnologia sem fazer seu uso; $9,1 \%(n=10)$ o desconheciam e apenas 5,5\% $(n=6)$ utilizavam o instrumento.

Ao abordar a experiência que os acadêmicos tiveram com o transiluminador cutâneo portátil, 66,7\% citaram ser vantajosa e 55,6\% relataram que seu uso contribui para a visualização das veias. Revisão integrativa da literatura com análise meticulosa de 25 artigos revelou que os profissionais que utilizam a transiluminação por luz de fibra ótica aumentam a taxa de sucesso global da cateterização periférica em comparação a utilização do método tradicional. Outrossim, o uso dessas tecnologias facilita a inserção de CVPs, promovendo a segurança e o maior tempo de permanência do dispositivo (Santos et al., 2020). 
Considerando-se o número de tentativas de punção, 33,3\% (n=3) realizaram a PVP na primeira tentativa com o transiluminador cutâneo portátil, enquanto $44,4 \%(n=4)$ dos acadêmicos tiveram o mesmo êxito sem o uso do aparelho. Contrapondo os dados da pesquisa, uma investigação realizada nos Estados Unidos com crianças, evidenciou que o número médio de tentativas foi maior no grupo com a técnica tradicional ( 4,2 tentativas) do que no grupo com a tecnologia $(2,6$ tentativas) com $\mathrm{p}<0,001$ (Kantor et al, 2016). Ao comparar o número de tentativas de PVP de enfermeiros que já tem experiência com a técnica, observou-se em estudo brasileiro que os profissionais que usaram a tecnologia e que acertaram a punção na primeira tentativa foi de $64,95 \%$ ( $n=63$ ), contra $82,52 \%(n=85)$ da técnica tradicional, com $p<0,005944$ (Oliveira et al., 2016).

Ressalta-se que o número de acertos pelos acadêmicos de enfermagem na primeira tentativa não foi significativo, pois ambos os grupos estavam realizando o procedimento de PVP pela primeira vez, e sabe-se que esta via requer habilidade técnica e prática. Vale destacar que para atingir maior proveito com o transiluminador cutâneo portátil, o profissional precisa estar familiarizado com ele, portanto, autores citam que para atingir sucesso e estar aptos ao uso são necessários de 20 a 25 punções com o uso da tecnologia, ou quatro meses de treinamento e para alcançar a competência, mais de 50 punções (Minami et al., 2012; Moore, 2014; Avelar et al., 2015; Ault et al., 2015).

Em relação a escolha das veias, percebe-se que a mediana foi a mais escolhida com $61,11 \%$ ( $n=11$ ), seguida da basílica e cefálica. Estes dados corroboram com o estudo de Yamagami \& Inoue (2019) em que as veias mais escolhidas para punção venosa com o uso da tecnologia foram as cefálicas, medianas e basílicas. Isso ocorre em virtude de elas serem mais calibrosas, portanto mais fáceis para a punção (Mukai et al., 2020). Também é importante frisar que em pacientes adultos, veias muito superficiais $(<0,3 \mathrm{~cm})$ e muito profundas $(>1,5 \mathrm{~cm})$ são difíceis de puncionar (Nakayama et al, 2020).

Identifica-se que para o uso seguro do transiluminador cutâneo portátil, faz-se necessário o desenvolvimento de novos treinamentos com os acadêmicos de enfermagem para que eles consigam adquirir habilidade e competência. Além do mais, conhecer os elementos que contribuem para o sucesso da punção com o uso da tecnologia garante uma técnica assertiva e com menos complicação ao paciente. Como limitação desta pesquisa, cita-se o pequeno número de acadêmicos de enfermagem participantes e de artigos que citam o uso de transiluminador cutâneo portátil para comparação dos resultados. O fato dos participantes que foram puncionados serem saudáveis somam-se às limitações, pois tal característica pode ter contribuído para o maior acerto na primeira tentativa para puncionar.

\section{Conclusão}

A experiência para os acadêmicos de enfermagem em relação à punção venosa periférica com o uso do transiluminador cutâneo portátil foi identificada como positiva, pois conseguiram visualizar a veia com mais facilidade, relataram que a prática foi válida ao aprendizado e foi uma experiência vantajosa. Entretanto, os acadêmicos que utilizaram essa tecnologia também citaram algumas dificuldades como puncionar a veia na primeira tentativa e consideraram difícil manipulação do aparelho.

\section{Referências}

Alves, D. A., Lucas, T. C., Martins, D. A., Cristianismo, R. S., Braga, E. V. O., \& Guedes, H. M. (2019). Avaliação das condutas de punção e manutenção do cateter intravenoso periférico. Revista de Enfermagem do Centro-Oeste Mineiro, 9: e3005. https://doi.org/10.19175/recom.v9i0.3005

Ault, M. J., Tanabe, R., \& Rosen, B. T. (2015). Peripheral intravenous access using ultrasound guidance: defining the learning curve. J Assoc Vasc Access, 20(1):32-6. https://doi.org/10.1016/j.java.2014.10.012

Avelar, A. F. M., Peterlini, M. A. S., Onofre, P. S. C., Pettengill, M. A. M., \& Pedreira, M. L. G. (2010). Training of nurses in the use of intravascular ultrasound in peripheral puncture. Acta Paul Enferm, 23(3):433-6. https://www.scielo.br/j/ape/a/7LsGSkJfMQzFRcvtnX43StS/?lang=en\&format=pdf 
Caramelo, A. C. L. M., Pereira, M. C. A. R. S., Branco, M. Z. P. C., dos Santos, C. A. G., \& Pires. P. M. R. P. (2019). A história da punção venosa e o cuidado de enfermagem. História da Ciência e Ensino: construindo interfaces, 20 (esp):89-96. https://doi.org/10.23925/2178-2911.2019v20espp89-96

Dougherty, L., Bravery, K., Gabriel, J., Kayley, J., Malster, M., Scales, K., \& Inwood, S. (2010). Standards for infusion therapy. Royal Collage of Nursing. https://ivtherapyathome.heartofengland.nhs.uk/wp-content/uploads/2013/05/RCN-Guidlines-for-IV-therapy.pdf.

Gorski, L., Hadaway, L., Hagle, M. E., McGoldrick, M., Orr, M., \& Doellman, D. Infusion therapy standards of practice. (2016). Journal of Infusion Nursing, 39(sup. 1): 1-160. https://source.yiboshi.com/20170417/1492425631944540325.pdf

Kantor, D. B., Su, E., Milliren, C. E., \& Conlon, T. W. (2016). Ultrasound guidance and other determinants of successful peripheral artery catheterization in critically ill children. Pediatr Crit Care Med, 17(12):1124-1130. https://doi.org/10.1097/PCC.0000000000000936

Marinho, A. M., Sabino, F. H. O., Monteiro, D. A. T., Filgueira, V. S. A., de Azevedo, G. N., \& Toffano, S. E. M. (2019). Difficult peripheral venous puncture in adults: integrative review. Rev Enferm UERJ, 27: e42567. https://doi.org/10.12957/reuerj.2019.42567

Minami, L. F., dos Santos, P. T., Ferrari, C. R. S., Ciampone, M. H. T., Messas, J. T., \& Mira, V. L. (2012). Avaliação do treinamento "Prevenção e tratamento de Úlcera por Pressão" ministrado à equipe de enfermagem. Rev. Eletr. Enf, 14(3):663-70. https://revistas.ufg.br/fen/article/view/16035/13439

Moore, C. L. (2014). Ultrasound first, second, and last for vascular access. J Ultrasound, 33(7):1135-42. https://doi.org/10.7863/ultra.33.7.1135

Mota, S. P., do Nascimento, J. S., Azedo, S. P. B. M., de Freitas, C. C. S., Feijão, A. R., \& Melo, G. S. M. (2019). Punção venosa periférica: análise dos registros de acadêmicos de enfermagem. Revista de Enfermagem da UFSM, 9(39):1-15. https://doi.org/10.5902/2179769230148

Mukai, K., Nakajima, Y., Nakano, T., Okuhira, M., Kasashima, A., Hayashi, R., Yamashita, M., Urai, T., \& Nakatani, T. (2020). Safety of Venipuncture Sites at the Cubital Fossa as Assessed by Ultrasonography. J Patient Saf, 16(1):98-105. https://doi.org/10.1097/PTS.0000000000000441

Nakayama, Y., Takeshita, J., Nakajima, Y., \& Shime, N. (2020). Ultrasound-guided peripheral vascular catheterization in pediatric patients: a narrative review. Crit Care, 24(1), 1-11. https://doi.org/10.1186/s13054-020-03305-7

O’grady, N., Alexander, M., Burns, L. A., Dellinger, E. P., Garland, J., Heard, S. O., Lipsett, P. A., Masur, H., Mermel, L. A., Pearson, M. L., Raad, I. I., Randolph, A. G., Rupp, M. E., Saint, S., \& Healthcare Infection Control Practices Advisory Committee (HICPAC). (2011). Summary of recommendations: Guidelines for the Prevention of Intravascular Catheter-related Infections. Clin Infect Dis, 52(9):162-193. https://doi.org/10.1093/cid/cir138

Oliveira, A. M., Danski, M. T. R., \& Pedrolo, E. (2016). Technological innovation for peripheral venipuncture: ultrasound training. Rev. Bras. Enferm, 69(6): 1052-8. https://doi.org/10.1590/0034-7167-2016-0125

Oliveira, A. S. S., Costa, P. J. S., Graveto, J. M. G. N., Costa, F. J. G., Osório, N. I. A., Cosme, A. S. T. C., \& Parreira, P. M. D. (2019). Práticas dos enfermeiros na cateterização intravenosa periférica: um estudo descritivo. Rev. Enf. Ref, 4(21):111-121. https://doi.org/10.12707/RIV19006

Potter, P., Stocket, P. A., Perry, A. G., Hall, A. M., \& Ostendorf, W. R. (2017). Fundamentos de Enfermagem. (9a ed.), Elsevier.

Rickard, C. M., Webster, J., Wallis, M. C., Marsh, N., McGrail, M. R., French, V., Foster, L., Gallagher, P., Gowardman, J. R., Zhang, L., McClymont, A., \& Whitby, M. (2012). Routine versus clinically indicated replacement of peripheral intravenous catheters: a randomized controlled equivalence trial. Lancet, 22;380(9847):1066-74. https://doi.org/10.1016/S0140-6736(12)61082-4.

Santos, L. M., Santos, S. A., Silva, B. S. M., de Santana, R. C. B., \& Avelar, A. F. M. (2020). Influência de tecnologias para avaliação/visualização vascular no cateterismo intravenoso periférico: Revisão integrativa. Esc. Anna Nery, 24(3): e20190355. https://doi.org/10.1590/2177-9465-EAN-2019-0355

Silva, M. C. N., \& Machado, M. H. (2020). Sistema de Saúde e Trabalho: desafios para a Enfermagem no Brasil. Ciência \& Saúde Coletiva, 25(1):7-13. https://doi.org/10.1590/1413-81232020251.27572019

Soares, G. L. (2018). Tecnologias semióticas em enfermagem clínica dermatológica, SC. Monografia (Graduação em Enfermagem - Centro de Ciências da Saúde. Universidade Federal de Santa Catarina, p. 147.

Yamagami, Y., \& Inoue, T. (2020). Patient Position Affects Venodilation for Peripheral Intravenous Cannulation. Biol. Res. Nurs, 22(2), 226-233. $10.1177 / 1099800419893027$ 